and other aspects of social organization. $\mathrm{He}$ is also collecting autobiographies and other personal documents. He arrived in Addis Ababa in October 1958, and after spending a few months there to consult with Ethiopian specialists, and to work on the language and in libraries and museums, proceeded to the Konso territory, where he will remain (with brief intervals in Addis Ababa) until the end of his stay in Ethiopia, in January 1960.

\title{
Création d'une Université à Tananarive
}

LE 8 juillet 1959, le Conseil Exécutif de la Communauté a décidé la création d'une Université à Tananarive pour le premier octobre $196 \mathrm{r}$.

Cette université proviendrait de la transformation de l'actuel Institut des Hautes Études qui comprend: une École de Droit; une École de Médecine et de Pharmacie; une École des Sciences; une École des Lettres; un Institut d'Études Judiciaires; un Institut d'Études Malgaches.

Actuellement, les études de Droit, de Sciences et de Lettres s'arrêtent après la licence. L'École de Médecine et de Pharmacie est embryonnaire, les étudiants ne pouvant accomplir à Tananarive que la première année de pharmacie (année de stage). L'Institut d'Études Judiciaires prépare les licenciés en Droit aux professions de magistrat et d'avocat. L'Institut d'Études Malgaches dispense à tous les étudiants un enseignement sur les problèmes physiques et humains de Madagascar. Il y a actuellement environ 450 étudiants à Tananarive, chiffre qui, dans les années prochaines, devrait augmenter à peu près de 100 par an. En 1959-60 sera vraisemblablement ouverte une École Malgache d'Administration. Les années qui viennent verront la création d'un Institut Technique et d'un Institut Agronomique et Vétérinaire, ainsi que le développement normal de l'École de Médecine et Pharmacie dès l'achèvement de l'hôpital universitaire.

\section{University Institute of Somalia: Inauguration and Symposium on International Co- operation}

THE inauguration of the University Institute of Somalia at Mogadishu on 12 January 1960 was followed by a Symposium on 'International Co-operation in Africa', to which representatives from a number of countries, as well as the international organizations working in Africa, were invited. The programme was divided into three parts: aims and means of international co-operation in Africa; activities of international organizations in Africa; and problems and results of international co-operation in the countries represented at the Symposium.

\section{Recherches d'Ethno-archéologie dans la République du Tchad (Io* Mission Lebeuf)}

ComposíE de Jean-Paul Lebeuf, Maître de Recherches au C.N.R.S., Collaborateur scientifique de l'Institut de Sociologie Solvay (Bruxelles), Annie M. D. Lebeuf, Attachée de Recherches au C.N.R.S., et de Néné-Khali Camara, Diplômé du Centre de Formation et de Recherches Ethnographiques du Musée de l'Homme, la mission a commencé ses travaux en décembre 1959.

Organisée avec l'appui du Centre National de la Recherche Scientifique, du Gouvernement de la République du Tchad et du Haut Commissariat près la République du Tchad, la mission a pour objectif essentiel la coordination des recherches de sciences humaines dans la région tchadienne.

Son programme se répartit comme suit: le repérage des sites anciens et l'établissement de la carte archéologique du Tchad (et du Cameroun) dans le cadre de l'Atlas préhistorique 